OPEN ACCESS

Edited by:

Ondrej Slaby,

Brno University of

Technology, Czechia

Reviewed by:

Anita Muthukaruppan,

The University of Auckland,

New Zealand

Arsheed A. Ganaie,

University of Minnesota Twin Cities,

United States

*Correspondence: Jingbo Wang

wangjingboqd2008@163.com

Furong Li

frli62@163.com

Specialty section: This article was submitted to

Cancer Genetics,

a section of the journal

Frontiers in Oncology

Received: 30 July 2019 Accepted: 13 March 2020

Published: 07 April 2020

Citation:

Wang J, Wang Y, Han L, Shahen M, Hu C and Li F (2020) Multi-Omics Integration Reveals a Competitive Endogenous RNAs Network for the Identification of Progression Biomarkers and the Stratification of

Patients Diagnosed With Nephroblastoma

Front. Oncol. 10:444

doi: 10.3389/fonc.2020.00444

\section{Multi-Omics Integration Reveals a Competitive Endogenous RNAs Network for the Identification of Progression Biomarkers and the Stratification of Patients Diagnosed With Nephroblastoma}

\author{
Jingbo Wang ${ }^{1,2 *}$, Yuan Wang ${ }^{3}$, Liang Han ${ }^{4}$, Mohamed Shahen ${ }^{5}$, Chaofeng $\mathrm{Hu}^{2}$ and \\ Furong $\mathrm{Li}^{1,2 *}$
}

\begin{abstract}
${ }^{1}$ Translational Medicine Collaborative Innovation Center, The Second Clinical Medical College (Shenzhen People's Hospital), Jinan University, Shenzhen, China, ${ }^{2}$ Integrated Chinese and Western Medicine Postdoctoral Research Station, Jinan University, Guangzhou, China, ${ }^{3}$ School of Nursing, Weinan Vocational and Technical College, Weinan, China, ${ }^{4}$ Department of Andrology, Fangshan Hospital, Beijing University of Chinese Medicine, Beijing, China, ${ }^{5}$ Zoology Department, Faculty of Science, Tanta University, Tanta, Egypt
\end{abstract}

Specific types of nephroblastoma (Wilms' tumor, WT) are known to associate with poor overall survival. Emerging experimental evidence has demonstrated that competitive endogenous RNA (ceRNA) networks have important roles in regulating cancer occurrence, but the roles of ceRNAs in regulating the WT progression and the patient outcomes remain unclear. Using the multi-omics data of $132 \mathrm{WT}$ patients collected from TARGET database, an integration analysis pipeline was performed to construct a highly reliable ceRNA network. As results, a total of 147 nodes (116 mRNAs, 15 miRNAs, and 16 IncRNAs) were identified and used to explore the underlying mechanism for WT progression. WGCNA analysis further identified several prognostic molecules, including hsa-mir-93, LINC00087 and RP5-1086K13, that significantly associated with the overall survival rate. And, enrichment analysis verified the participation of these molecules in tumor-related pathways, such as those controlling autophagy and cadherin-mediated adhesion. Importantly, the WT patients were classified into three categories according to the ceRNA network, which significantly correlated with the overall survival. In conclusion, the ceRNA network could be a promising tool to further validate the prognostic biomarkers and categories of patients diagnosed with WT.

Keywords: nephroblastoma, competitive endogenous RNA, tumor progression, prognostic marker, tumor categories

\section{INTRODUCTION}

Nephroblastoma, also known as Wilms' tumor (WT), is a complex childhood tumor of the kidney and the most prevalent type of kidney cancer in children (1). It is the underlying cause of 6-14\% of children with tumors and up to $95 \%$ of all kidney cancers in children, affecting approximately one child per 10,000 worldwide under 15 years of age (2). Multiple effective treatments have been 
developed, and the overall survival rate of WT has reached $90 \%$ within the past several decades (3). However, recurrence still occurs in $\sim 15 \%$ of WT patients with favorable pathological findings and practically $50 \%$ of anaplastic WT patients. Relapsed patients, as well as those with bilateral or unilateral high-risk tumors, who are at great risk for significant late effects of therapy, continue to have poor event free survival rates (4-6). Therefore, to develop novel and effective therapeutic approaches, it is important to understand the eventual downturn in the disease state that many WT patients will undergo.

Carcinogenesis is a complex process driven by abnormal gene combinations which may vary greatly between patients. WT is a genetically heterogeneous disease. Several gene alterations in predisposing loci have been well-identified, including mutations in Wilms' tumor gene 1 (WT1), catenin beta 1 (CTNNB1), insulin-like growth factor-2 (IGF2) and Wilms' tumor gene on the X chromosome (WTX) (7-9). The multifaceted regulation across multiple genes is important for the development of this disease. Numerous studies have reported that non-coding RNAs, including microRNAs (miRNAs) and long non-coding RNAs (lncRNAs), may play a critical role in cancer development (10, 11). Approximately $15 \%$ of patients diagnosed with WT have mutations in the miRNA-processing genes (12). Gong et al. analyzed the miRNA expression profiles of WT patients and identified 5 potential prognosis biomarker (13). Another study using microarrays to perform miRNA and gene expression profiles of WT patients and found the relationship between abnormally expressed miRNA and tumor progress (14). Previous studies have focus on the single omics and research on transcriptome has been largely limited to mRNA. As previously discussed, a single factor cannot explain the development of cancer. However, computational analysis provides a good way to understand the interactions between different factors that may contribute to cancer development.

Recent studies have demonstrated another layer of miRNAmediated regulation that involves direct interactions between RNA molecules and common miRNAs. These RNAs, which were first presented in 2011 by Rubio-Somoza et al. (15), are known as competing endogenous RNAs (ceRNAs). The ceRNA hypothesis states that the pool of mRNAs, IncRNAs and other ncRNAs shares common microRNA response elements (MREs) with miRNAs, which can inhibit normal miRNA functions through competitive binding, thereby participating in the regulation of cell behavior (16). A recent bioinformatics study revealed that AFA-P1-AS1 acts as a ceRNA, competitively binding with miR423-5p and directly regulating genes in the Rho/Rac pathway, thereby enhancing nasopharyngeal carcinoma cell migration and invasion (17). Another study characterized a ceRNA network that could distinguish the mesenchymal subtype from other glioblastoma subtypes (18). Taken collectively, these findings support the usefulness of the ceRNA network in understanding the development of this disease.

Most recent studies on the ceRNA network consider miRNAs to be gene regulators that act alone, and they ignore the influence of other regulatory factors on the ceRNA network, such as epigenetic factors, transcription factors and gene copy number variation factors (19). Losing sight of these critical factors may lead to spurious miRNA-gene interactions, which may cause false positive results in the ceRNA network. The recently proposed Cancerin algorithm overcomes this problem by integrating muti-omic data (20). Another problem, however, involves the identification and characterization of suitable candidate nodes to expand the ceRNA network. Most studies have used differential expression patterns as a screening standard. However, due to the inherent characteristics of transcriptomic data, there is usually severe noise in the differentially expressed genes (21). In addition, the genes with the greatest variations are not necessarily the genes responsible for the phenotypic changes because of the complex hierarchical relationships within the biological regulatory network. Therefore, it is critical to identify the transcripts most associated with cancer progression and to define them as nodes in the expansion of the ceRNA network. Weighted Gene Co-expression Network Analysis (WGCNA) solves this problem well (22). This method has been widely used to screen the gene co-expression module and the key node, collectively known as the hub node, which is most closely related to phenotypic changes.

A fundamental approach to study the heterogeneity of WT is to stratify the patients according to the molecular characteristics. Thus, tumors can be divided into clinically and biologically meaningful subtypes $(23,24)$, with each subtype associating with similar molecular markers. Previously, many attempts have been made to stratify various cancers using high quality transcriptomic signatures, and some molecular subtypes in breast cancer have now been clinically validated such as Mammaprint and Oncotype Dx (25). Since the endogenous RNA network integrates multiple layers of information, we hypothesized that the classification of patients using the ceRNA network would be useful.

In this study, we obtained RNA-seq, copy number and methylation data from the TARGET Database of 132 WT patients at different stages of the disease. The candidate miRNAs, mRNAs and $\operatorname{lncRNAs}$ related to tumor progression were identified by co-expression analysis. Furthermore, multi-omics data (genome, transcriptome, and epigenome) were integrated, and different methods were adopted to build a high-confidence ceRNA network. As a result, several key lncRNAs, which could predict patient prognosis, were identified and further investigated. According to these results, six lncRNAs could be used as reliable indicators of patient prognosis. In addition, we stratified patients using the condense cluster method and divided them into subtypes with significant clinical significance based on the ceRNA network (Figure S1). In summary, the ceRNA network obtained from this multi-group study represents an effective approach to study the stratification of patients, as well as to identify the mechanism responsible for the progression of WT.

\section{MATERIALS AND METHODS}

\section{Patients and TARGET Data Retrieval}

The clinical data of 132 WT patients were obtained from TARGET (Therapeutically Applicable Research To Generate Effective Treatments) database. The survival and stage information were included in this database. The RNA-seq, miRNA-seq, DNA methylation and DNA copy number information were also downloaded. Patients with incomplete clinical information were filtered out, and $132 \mathrm{WT}$ patients were 
retained. The study is in accordance with publication guidelines provided by TARGET (https://ocg.cancer.gov/programs/target/ target-publication-guidelines). Since the data comes from the TARGET database, no further approval was required from the Ethics Committee.

\section{RNA Sequence Data Processing}

The RNA FPKM (fragments per kilobase of exon per million fragments mapped) and miRNA expression data of $128 \mathrm{WT}$ patients were obtained from TARGET database. All data from the samples were derived from the Illumina Hi-Seq platform and freely available to download. Furthermore, "annotable" package based on the R environment was used to distinguish the lncRNA and coding RNA. Finally, 20,347 mRNA, $5983 \operatorname{lncRNA}$ and 1870 miRNA were identified.

\section{Copy Number Alteration}

Mean copy numbers of chromosomal segments in the whole genome were provided by level 3 copy number alteration data from TARGET. Using the genomic location information of protein coding genes provided by GENCODE Release 26 (GRCh37), the R Bioconductor package CNTools were applied to transform the segmented CNA data into a gene-level data matrix where each entry represented copy number value of a gene in a definite sample.

\section{Methylation Data Pretreatment}

The genome-wide methylation level of $\sim 450,000 \mathrm{CpG}$ sites needed to be measured in level 3 DNA methylation data from TARGET samples. The ratio of methylated probe intensity of the overall intensity (sum of methylated and unmethylated probe intensities) was defined as the methylation level of each $\mathrm{CpG}$ site (i.e., $\beta$ value). Thus, $\beta$ ranges between 0 and 1 , with 0 being hypomethylated and 1 being hypermethylated. Previous research indicated that the methylation of $\mathrm{CpG}$ sites in the promoter regions resulted in gene expression change. Therefore, only considered $\beta$ values of $\mathrm{CpG}$ sites are in genes' promoter regions. Thus, Bioconductor annotation package AnnotationHub was used to identify the probes positioned at the upstream 2001,500 base pairs from the gene transcription start site. Gene's methylation level was estimated as the mean of its associated upstream probes' $\beta$ values.

\section{Identification of Differentially Expressed Genes, miRNAs and IncRNAs}

To identify mRNA, lncRNA, and miRNA which was associated with tumor progression, we divided the tumor samples into 2 groups (early stage and advanced stage) and used LIMMA package ( $\mathrm{R}$ version 3.4.1) to analyze differences in the expression levels between the two tumor groups. As the raw transcriptomic data may be noisy, several filtering processes were performed to improve the quality of the expression profile. Firstly, we removed RNAs with low-expression values in more than $70 \%$ of the WT patients. Then, we calculated the coefficient of variation (CV) in gene expression for each RNA, and remove the $20 \%$ of RNAs with the lowest $\mathrm{CV}$ values. In addition, for multiple ensemble gene ids corresponded to the same gene symbol, the genes with maximum $\mathrm{CV}$ was retained to represent that gene. The differentially expressed mRNAs, IncRNAs and miRNAs were identified based on the same thresholds (absolute $\log 2 \mathrm{FC}>2.0$ or $p<0.05$ ).

For immunologic gene sets, we first captured relevant microarray datasets published in the immunology literature that has raw data deposited to Gene Expression Omnibus (GEO) with accession number GSE37301, GSE37605, GSE6259, and GSE2405. These studies included both human and mice data. However, it is proved that the characteristics of the activation of lymphocytes and differentiation of bone marrow cells were highly conserved between human and mouse cells (26). More importantly, instead of focusing on the changes of individual genes, we used the enrichment analysis to determine the overall coincidence degree of WT deterioration related genes and immune-related gene sets, which helped us identify the biological significance of different genes. For each published study, the relevant comparisons were identified (e.g., WT vs. KO; pre- vs. post-treatment etc.) and brief, biologically meaningful descriptions were created. All data were processed and normalized the same way to identify the gene sets, which correspond to the top or bottom genes (FDR $<0.02$ or maximum of 200 genes) ranked by mutual information for each assigned comparison.

\section{Screening of Candidate Genes}

Based on the previously identified mRNA, IncRNA, and miRNA, WGCNA was carried out to acquire candidate mRNA, lncRNA, and miRNA which is relative to clinical stages of the disease. The correlation of gene expression profile with module eigengenes (Mes) was defined as the module membership (MM) and the correlation between gene and external traits was defined as gene significance (GS) measure. The genes with $|\mathrm{GS}+\mathrm{MM}|$ ranked above the top $10 \%$ were selected as candidate gene together with genes in the core module.

\section{Functional Enrichment Analysis of Core Module Gene}

For a deeper understanding of the biological effects and pathways of the aberrantly expression core module gene, Gene Ontology (GO) Biological Process, Kyoto Encyclopedia of Genes, and Genomes (KEGG) pathway analyses were constructed using the R/Bioconductor package of Clusteprofiler. Functional enrichment analysis was based on the threshold of $P<0.05$. The predicted function of IncRNA is based on the function of the gene with the highest correlation with lncRNA in ceRNA network.

\section{Construction of ceRNA Network}

In order to get a reliable ceRNA network, the candidate mRNA, miRNA and lncRNA which was selected in part 6 was invoked as nodes. Cancerin and GDCRNATools were utilized to construct the tumor deterioration-related ceRNA network. Cancerin integrated multidimensional cancer genomics data in order to infer cancer-associated ceRNA interaction 
networks which could identify the miRNAs contributed to the differential expression of RNA's. The newly developed GDCRNATools is used for deciphering the lncRNA-mRNA related ceRNA regulatory network as well as many routine analysis including functional enrichment analysis, DEG analysis and survival analysis in cancers. The code provided by Cancerin was used to integrate transcriptome, DNA methylation and copy number information to predict ceRNA network. Furthermore, ceRNA network was also predicted with GDCRNATools. Finally, union of the two ceRNA networks was used as the ceRNA network.

\section{Patient Stratification}

In order to affirm patient stratification based on ceRNA network, a patient similarity matrix was constructed. Each element in the matrix represents the correlation coefficient based on ceRNA network. Then, the ConsensusClusterPlus package was used to reclassify patients. Furthermore, the similarity between samples was calculated by Pearson's correlation. Samples were distributed in $\mathrm{k}$ clusters by the PAM algorithm. The best number of clusters was determined by relative change in area under the CDF (Consensus Cumulative Distribution Function) curve compared $\mathrm{k}$ and k-1 (27).

\section{Survival Analysis}

Survival analysis for all RNAs in the ceRNA network was carried out by using the $\mathrm{R}$ survival package (https://CRAN.R-project. org/package=survival, Version: 2.41-3). The log-rank test was carried out to identify whether the expression of lncRNAs, mRNAs and miRNAs was correlated with overall survival. For the overall survival rates, we use the log-rank test to compare the significant differences in univariate analysis between each subgroup. Unless otherwise specified, a $P<0.05$ is considered as statistically significant.

\section{Data Availability}

The datasets analyzed during the current study are available in the TARGET repository (https://ocg.cancer.gov/programs/ target/data-matrix). All relevant data are within the paper and its Supporting Information files.

\section{RESULTS}

\section{Gene Expression Patterns in WT Patients at Different Stages of the Disease}

The clinical data of 132 WT patients at different stages of the disease were downloaded from the TARGET Database, including

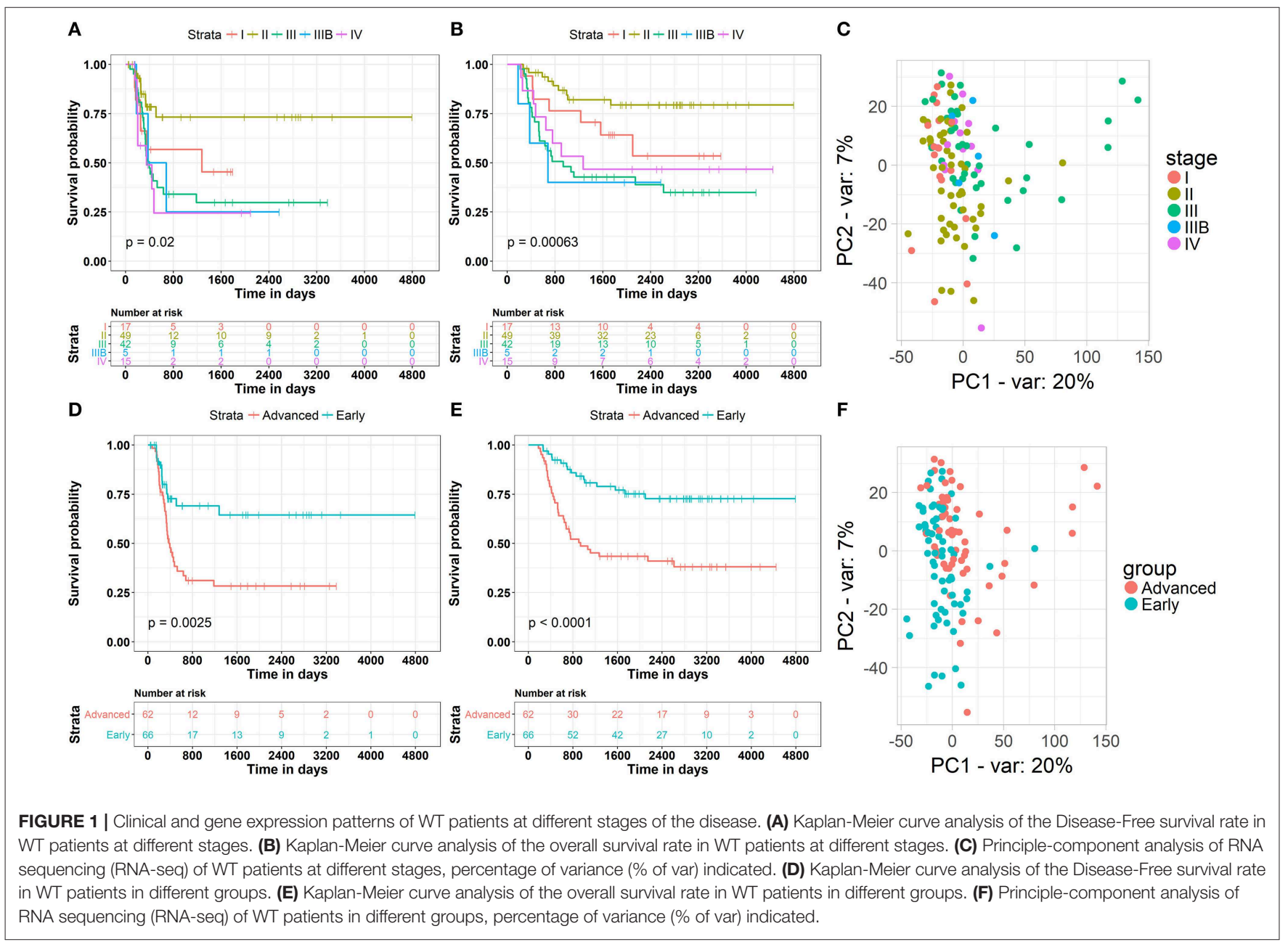


progression-free survival, total survival, disease stage, and the corresponding RNA-seq data. The relationship between patient survival and disease stage, which was first examined in our study, indicated that the traditional stage significantly correlated with the patient disease-free survival and overall survival rates (Figures 1A,B). Surprisingly, the worst disease-free survival rate was not observed in patients at the latest stage of the disease. For example, we found by disease-free survival analysis that patients at stage II were better than those at stage I, and similar results were observed by overall survival analysis. The most likely cause of this result is that there are a few samples of this tumor and fewer clinical samples have been collected, resulting in some deviations in survival statistics. Another possibility is that due to the difficulty in early diagnosis of cancer, some patients were found in stage I, and in fact the tumor development was very close to stage II, and even worse than the normal stage II patients in terms of partial molecular expression. Principal component analysis (PCA) was used to reduce the dimensionality of the gene expression data and to visualize two components on the scatter plot. The gene expression data of patients at different stages of the disease did not significantly cluster (Figure 1C). This may have been due to the fact that conventional disease stages tend to focus on phenotypes, such as tumor size and metastasis, while ignoring genotypes and molecular mechanisms, which may underlie ineffective treatments. Considering the contradiction between the survival rate of patients and the early stages of the disease, and the difficulty in discerning the survival rate and the late stages of the disease, we first classified phase I and II patients as "early," whereas the remaining patients were classified as "advanced" to obtain information on the key biomolecules involved in cancer progression. According to results from survival analysis, "early' patients were far superior to "advanced" patients (Figures 1D,E) in both the disease-free progression survival rate and the total survival rate. More importantly, PCA analysis showed that patients at an "early" stage presented different patterns of gene expression than those at an "advanced" stage (Figure 1F), suggesting that several key molecules are involved in the progression of renal myoblastoma. These results lay the foundation for the search of biomarkers associated with progression.

\section{Clinical Relevant Candidate Nodes Identification}

To identify candidate molecules associating with the eventual downturn and poor prognosis of nephroblastoma, WGCNA was used to analyze the co-expression network of mRNA-miRNA-lncRNA. First of all, a differentially expressed gene was defined as a gene whose $\log 2$ value of the fold change of the expression $(\log F C$ ) was $>2$ and the $P<0.05$. We identified 4,285 differentially expressed mRNAs and 246 differentially expressed lncRNAs using the LIMMA R Package. The differentially expressed RNAs are listed in Table S1. We used a similar procedure and identical criteria to screen the differentially expressed miRNAs and identified 259 differentially expressed miRNAs (Table S2).
Based on these differentially expressed mRNAs, miRNAs and lncRNAs, we analyzed their co-expression networks using WGCNA to identify the most relevant molecular modules associating with clinical decline. Soft-threshold beta was selected as a suitable weighted parameter of the adjacency function before constructing the weighted co-expression network. After performing the calculation, we selected the correlation coefficient closest to 0.8 (soft-threshold catcher $=4$ ) to construct the gene modules using WGCNA (Figures 2A,B). After determining the soft threshold, all differentially expressed molecules (mRNAs, miRNAs and lncRNAs) were used to construct the weighted gene co-expression network. We found that the degree of node conformed to the power law distribution (Figures 2C,D).

Furthermore, 15 modules were identified through business linkage hierarchical clustering (Figure 3A). The red module was found to have the highest association with tumor progression $\left(R^{2}=0.42, P<0.01\right)$ (Figure 3B), and this module was selected as a clinically significant gene set for further analysis. To avoid missing other key genes related to tumor progression, highly expressed molecules of module membership (MM) and gene significance (GS) were added to the candidate node collection. We selected mRNAs, miRNAs and lncRNAs with cutoff criteria $(|\mathrm{MM}+\mathrm{GS}|$ ranked above top 10\%). After integrating the molecules in the previously established core modules, we

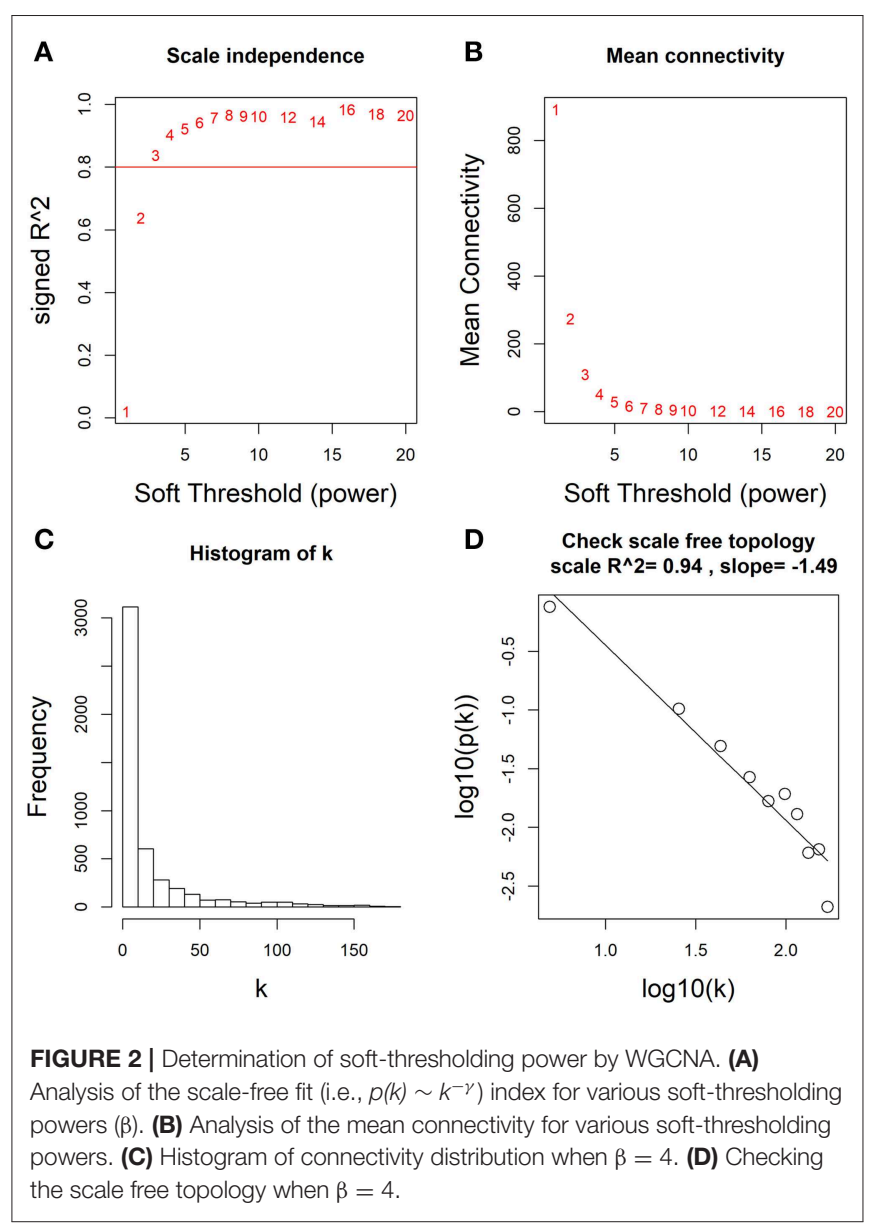




\section{A}

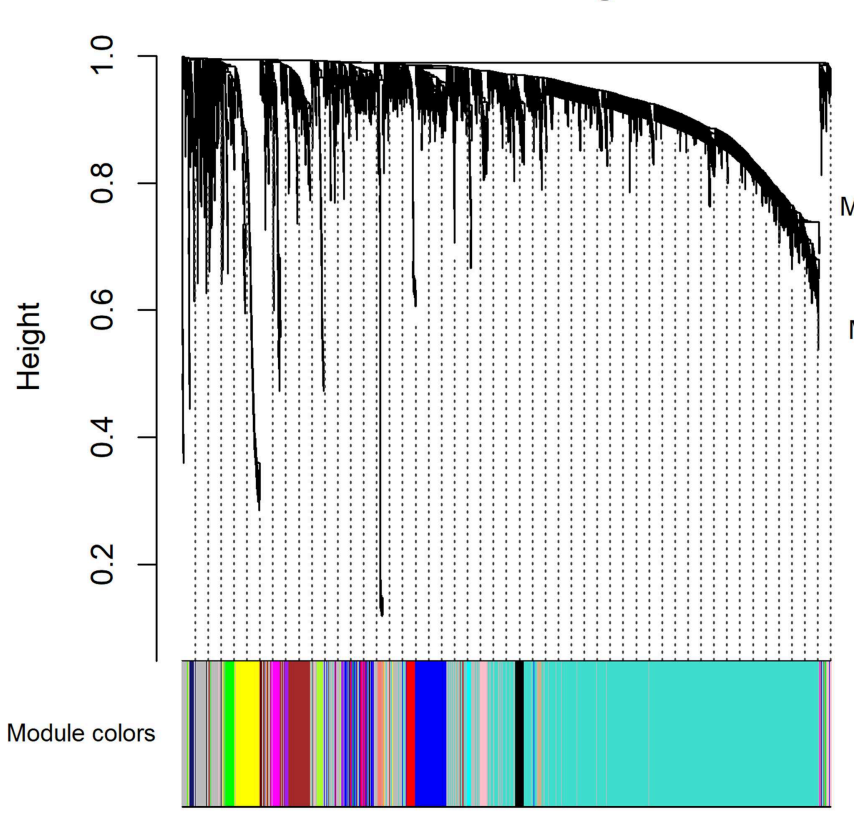

B

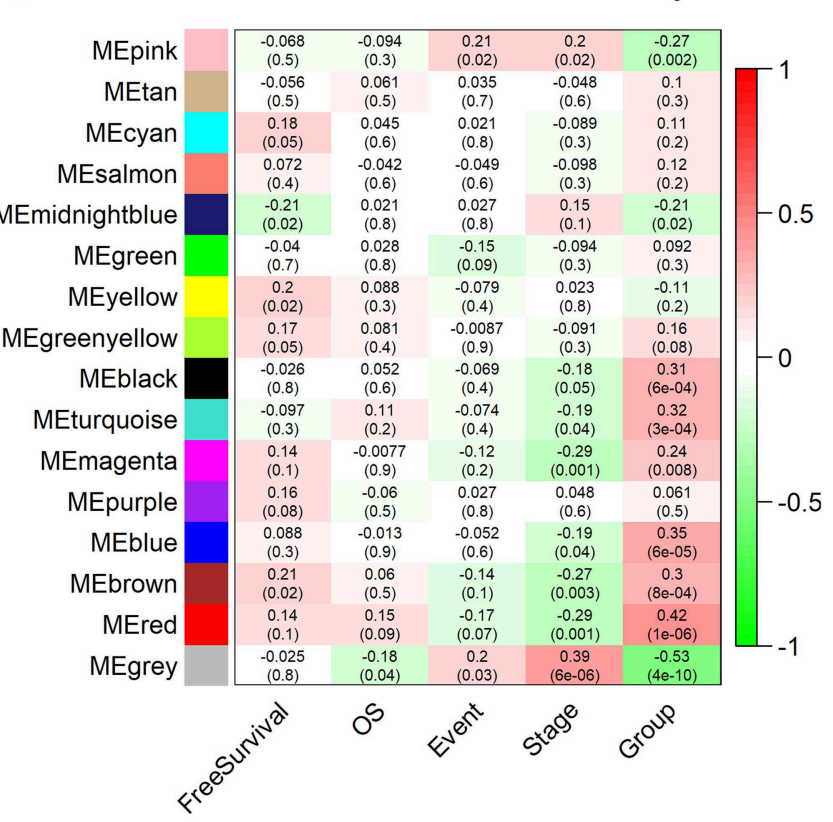

FIGURE 3 | Identification of the modules associating with the clinical traits of WT patients. (A) Dendrogram of all differentially expressed RNAs clustered based on a dissimilarity measure (1-TOM). (B) Heatmap of the correlation between module eigengenes and clinical traits of WT patients. "Event" is binary value indicating the death statue of each patient ( 0 for alive and 1 for dead), and "Group" represents the progression status of each patient ( 0 for early and 1 for advance).

obtained 458 mNRAs, 26 miNRAs, and 33 lncNRAs as the final molecule nodes to build the ceRNA network. The complete list of candidate molecules is presented in Table S3.

To explore the biological relevance between the candidate genes and clinical decline, Gene Ontology (GO) function and pathway enrichment analyses were performed using the $\mathrm{R}$ clusterProfiler Package (28) (Table S4). The results showed that the functions of the candidate genes mainly concentrated in areas of cell adhesion and cell fate specification (Figure 4A), which may ultimately contribute to cancer progression. As the immune regulation controls clinical development, we obtain immunologic gene sets from microarray gene expression data from immunologic studies and investigate whether our candidate genes are related to the innate immune system (Figure S2). In addition, we found that many immune-based mechanisms were related to the poor prognosis of WT, including the up-regulation of B cell-T cell interactions and foxp3 fusion (Figure S3).

\section{Construction of High Confidence ceRNA Competition Network}

To obtain a comprehensive and highly reliable ceRNA competitive network, we combined the results of two newly published ceRNA network prediction methods, namely, Cancerin and GDCRNAtools. Cancerin incorporates multiomics information from patients, including epigenetic, genomic and transcriptomic information, to increase the reliability of the network. In this study, miRNA-mRNA and miRNA-lncRNA relations with media confidence $<0$ were selected as the interactive relations of the ceRNA network. The parameter "media confidence" is derived from the Cancerin to filter the high reliable miRNA-mRNA and miRNA-lncRNA associations. Briefly, Cancerin estimated the confidence intervals of the correlation coefficient between one miRNA and each of its targets using LASSO regression for 500 time, and the median of the mid-point points of the 500 confidence intervals was defined as media confidence. And, "media confidence $<0$ " reflects reliable negative correlation relationship between the expression of a miRNA and its target. According to this method, 122 miRNA-target interactions and 64 nodes, including 39 mRNAs, 20 miNRAs and 5 lncRNAs, were selected (Table S5). In addition, we predicted 103 nodes and 330 edges in the ceRNA network using GDCRNA tools (Table S6). After combining these two sets of results, we constructed the endogenous competitive network of mRNA-miRNA-lncRNA (Figure 5, Table S7). This network contained 15 miRNAs, 16 lncRNAs, 116 mRNAs, and 407 interactions. The topological properties of the nodes in the network were further analyzed, and we found that hsa-miR-93 had the highest degree (degree $=58$ ), which represents the number of targets it interacts with. We investigated the relationship between this miRNA and patient survival (Figure 6A) and confirmed the significant correlation between has-mir-93 and the overall survival $(p<0.05)$. In addition, we investigated the relationship between other nodes in the network and patient survival, and found that $39.46 \%$ of the nodes correlated with patient survival ( $p<0.05$, Table S3).

LncRNAs regulate the expression of mRNAs through their interactions with miRNAs in the ceRNA network. Thus, the functions of lncRNAs can be reflected through the 


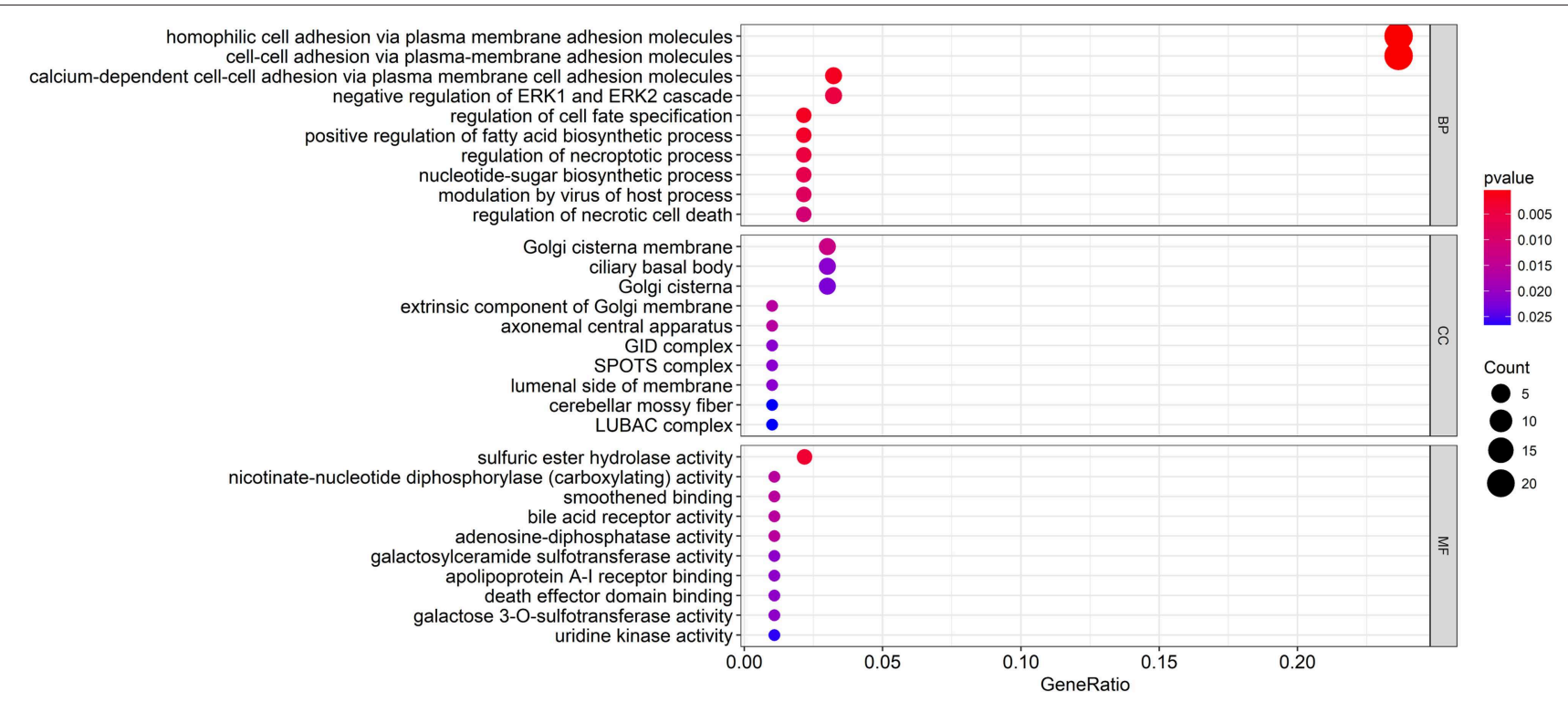

FIGURE 4 | Functional enrichment analysis of the candidate genes. The GO terms with the most significant $p$-values. The x-axis represents the number or gene ratio of core module mRNAs involved in the enrichment terms.

mRNAs regulated by them. In this study, two prognostic lncRNAs, LINC00087 and RP5-1086K13, were chosen for enrichment validation. We conducted GO enrichment analysis (GO_BP: biological processes, GO_CC: cellular component and GO_MF: molecular function) of their regulated mRNAs, and found that LINC00087 was mainly involved in autophagy and cadherin binding (Figure 7). RP5-1086K13 was involved in autophagosome dynamics and MAPKKK activity (Figure 8).

\section{Patient Stratification Based on the Clinically Relevant ceRNA Network}

These results revealed that the ceRNA network was useful in the identification of the prognostic biomarkers for WT. They also implied a correlation between the ceRNA network and the patient's clinical prognosis. Therefore, the subtypes of the patients based on this ceRNA network were further investigated. We first extracted the expression information for each node involved in the ceRNA network of each patient. Partitioning around methods (PAM)-based consensus clustering, followed by cluster reliability analysis (Methods), was used to investigate the case of dividing patients into $\mathrm{k}(k=2,3,4,5,6)$. Figure 9B shows the relative change in the area under the consensus cumulative distribution function (CDF) curve by comparing $\mathrm{k}$ with $\mathrm{k}-1$. According to these results, we found that the patients were classified into three categories using optimal classification standards, and the patients presented a relatively obvious clustering pattern (Figures 9A,B). To confirm whether our classification had clinical significance, the relationship between the new staging and the total survival were calculated. We found that there was a significant correlation between the staging of patients based on the ceRNA network and the total survival (Figure 9C, $p<0.05$ ).

\section{DISCUSSION}

The identification of genes and molecules that are related to tumor staging and progression is important for our understanding and control of the disease. WT is responsible for $\sim 95 \%$ of kidney tumors in children. Although improved therapies and prognosis methods have greatly increased the survival rate, additional effort is still needed to deal with the disease since $50 \%$ of children who relapse go on to die (29). In this study, a multidimensional network was constructed to provide new insights on the mechanism underlying the progression of WT.

The relationship between the survival rate and the histopathological grades was the first consideration in our study, and the general trend is that higher grades were significantly associated with a lower overall survival rate. However, according to the result of PCA, no distinct RNA expression patterns were observed in different grades (Figure 1C), indicating that the histological grades in this cohort may not be sufficient to represent patients with subtypes for further deciphering the molecular mechanism of WT progression. Taking these contradictions into consideration, we divided WT patients into "early" and "advanced" stages, and identified the different gene expression patterns between the two stages (Figures 1D-F). Because the progression-free survival and overall survival rates of "early" patients were far superior to those of "advanced" patients, it is possible that key biomolecules exist to influence the clinical decline of patients diagnosed with WT. To confirm the candidate molecules related to this process, WGCNA was carried out to construct a weighted co-expression network (Figures 2A,B). Recent research by Meng et al. which employed WGCNA, identified major genes that mediate immune cell activation and mitosis (30). Another WGCNA study by Guo 


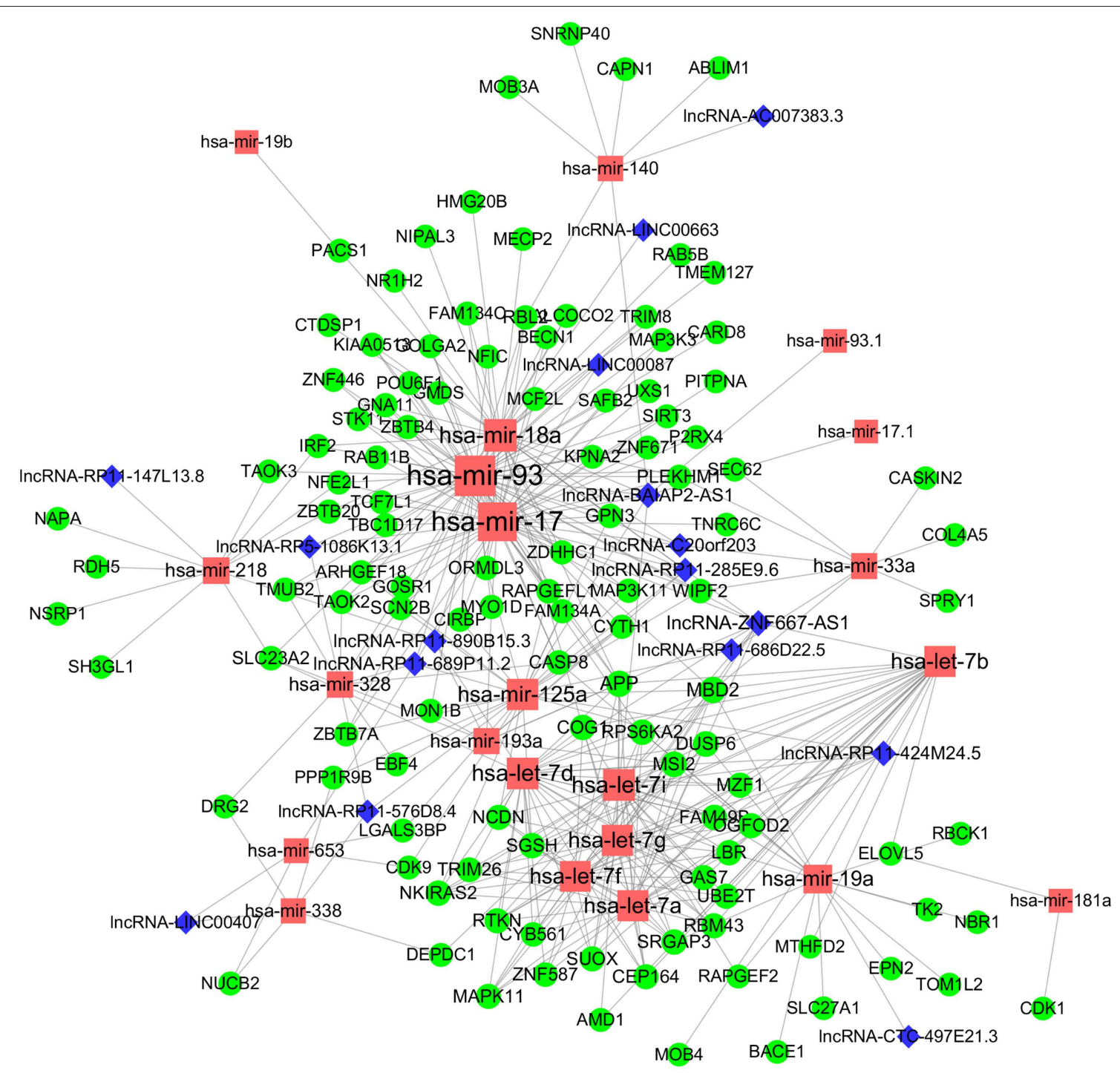

FIGURE 5 | CeRNA regulatory network in WT. The nodes highlighted in red indicate candidate miRNAs, the nodes highlighted in blue indicate candidate IncRNAs, and the nodes highlighted in green indicate candidate mRNAs. The size of the point represents the connectivity of the node, that is, the number of other points connected. Edge represents an interaction between two nodes.

et al. disclosed key genes with aberrant expression levels that may participate in the pathogenesis of head and neck squamous cell carcinoma (31). WGCNA has also been used to identify potential prognostic markers for uveal melanoma, Glioblastoma Multiforme, lung cancer, bladder cancer and so on (32-35). While for WT, WGCNA analysis has been used in one research to identify hub genes associated with high-risk pathogenesis (36). These results indicate that this method can be used to identify new candidate nodes in the ceRNA network. After constructing this co-expression network, we found that it was a scale-free network, which conforms to conventional biological network features (Figures 2C,D). Furthermore, molecules with high MM and GS were considered as node molecules, as well as previously identified modules. The network, which was constructed based on all the nodes, played an important role in the understanding the mechanism underlying the clinical decline of WT patients.

To further understand the relationship between these candidate molecules and WT progression, functional enrichment analysis was performed, and the results indicated that the functions mainly concentrated in areas such as cell adhesion, the ERK pathway and necrosis (Figure 4). As previously described, cell adhesion plays a major role in tumor biology. Research carried out by Liu et al. showed that the high expression of the focal adhesion protein kindlin-2 in solid tumors, as a prognostic biomarker, may indicate poor outcome in patients (37). Cellular glycosylation, which participates in cell-cell recognition, communication and adhesion, has a major impact on the acquisition of malignant characteristics 


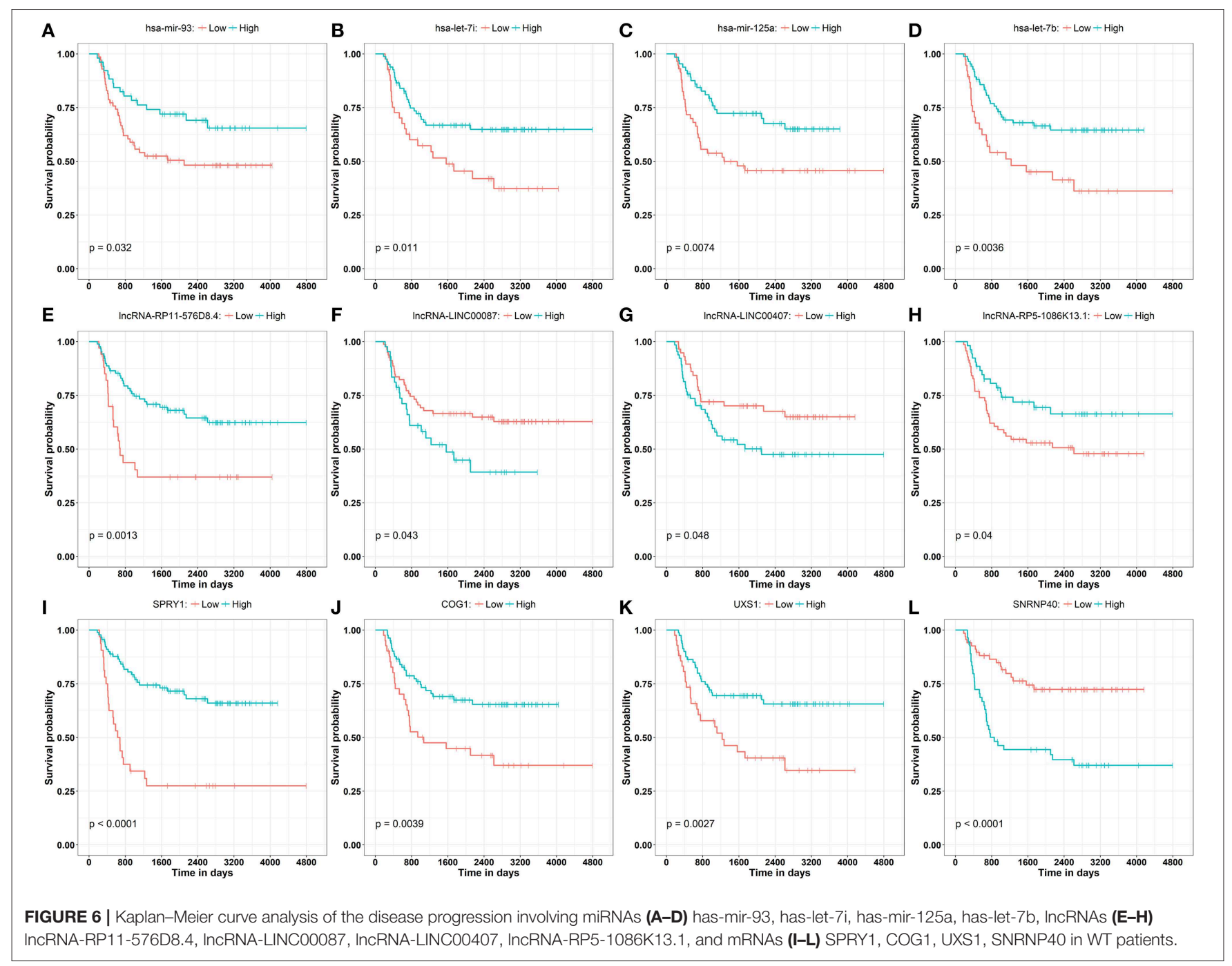

of gastric carcinoma (38). On the other hand, the cell surface CD56 glycoprotein, which controls cell adhesion and signaling, acts as a key biomarker in WT stem and progenitor cells (39). Another study demonstrated that ERK signaling may contribute to WT development (40). These results clearly support the reliability of our network analysis. Because interactions between the tumor and the immune system are also known to influence tumor progression, we analyzed the relationships between candidate genes and the immune system. We found that most genes were enriched in the innate immune system. We further explored the detailed immune-based functions, and found an up-regulation in Treg activity by our candidate genes (Figure 4C). An immunosuppressive microenvironment is essential for tumor progression, and Treg is an important supporter of this environment (41). Interestingly, a more pronounced Treginduced cytokine response was observed in WT patients, according to previous studies. To reverse the Treg activation induced by the candidate biomolecules may prevent the clinical decline of WT patients.
In this study, Cancerin and GDCRNAtools were combined to construct a superior confidence ceRNA competitive network (Figure 5). Among all nodes in this network, hsamiR-93 had the highest degree, and it was significantly related to the overall survival of patients (Figure 6A). Previous studies have already reported exosomal miR93 to be a novel biomarker for both the diagnosis and prognosis of hepatocellular carcinoma and triple negative breast cancer $(42,43)$. However, there were few studies addressing its roles in WT patients. Our results indicated that hsa-miR-93 may be defined as a prognostic biomarker of this disease.

In the ceRNA network, the competitive binding of mRNAs and lncRNAs to miRNAs can regulate mRNA expression. Therefore, we carried out enrichment analysis of mRNAs regulated by $\operatorname{lncRNAs}$ to reflect the function of $\operatorname{lncRNAs}$. LINC00087 and RP5-1086K13 were selected as prognostic biomarkers. LINC00087 is involved in autophagy and cadherin binding, whereas RP5-1086K13 is mainly involved in autophagosome dynamics and MAPKKK activity (Figures 7, 


\section{IncRNA-LINC00087 Function}

GO:0071467 cellular response to $\mathrm{pH}$ GO:2000377 regulation of reactive oxygen species metabolic process GO:0009268 response to $\mathrm{pH}$ GO:0006914 autophagy GO:0061919 process utilizing autophagic mechanism GO:0061919 process utilizing autophagic mechanism
GO:2000378 negative regulation of reactive oxygen species metabolic process
GO:0010611 regulation of cardiac muscle hypertrophy GO:0014743 regulation of muscle hypertrophy GO:0072593 reactive oxygen species metabolic process GO:0010507 negative regulation of autophagy GO:0005776 autophagosome GO:0045335 phagocytic vesicle
GO:0055037 recycling endosome GO:0005639 integral component of nuclear inner membrane-
GO:0031229 intrinsic component of nuclear inner membrane-
GO:0031300 intrinsic component of organelle membraneGO:0044453 nuclear membrane part GO:0005942 phosphatidylinositol 3-kinase complex GO:0098563 intrinsic component of synaptic vesicle membrane GO:0000137 Golgi cis cisterna
GO:0045296 cadherin binding GO:0061608 nuclear import signal receptor activity GO:0050839 cell adhesion molecule binding GO:0070403 NAD+ binding GO:0017136 NAD-dependent histone deacetylase activity GO:0030275 LRR domain binding GO:0031489 myosin $V$ binding GO:0034979 NAD-dependent protein deacetylase activity GO:0140142 nucleocytoplasmic carrier activity GO:0031683 G-protein beta/gamma-subunit complex binding

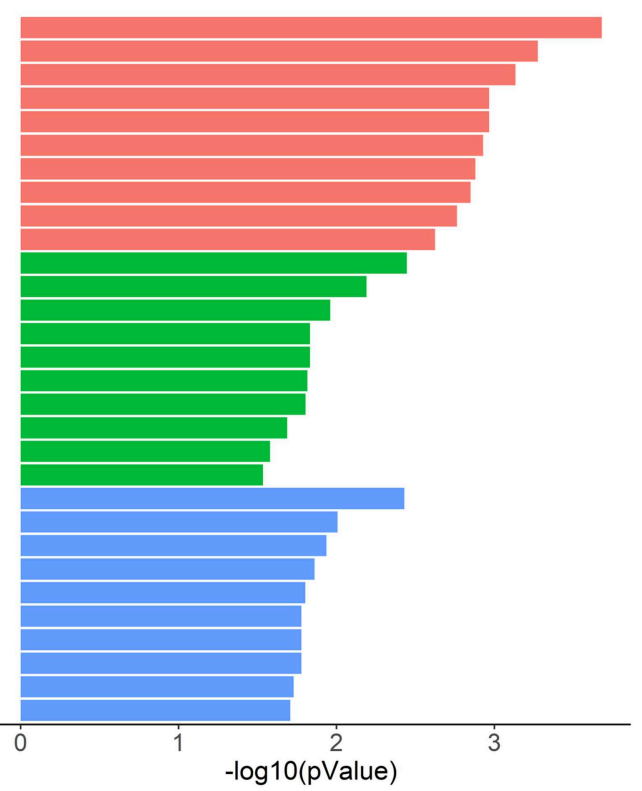

FIGURE 7 | Gene ontology enrichment analysis of LINC00087-regulated genes. The x-axis represents the -log10 ( $P$-value) of the enrichment analysis. Red, green and blue represent the enrichment of Gene Ontology (GO) Biological Process, Cellular component, and Molecular function, respectively.

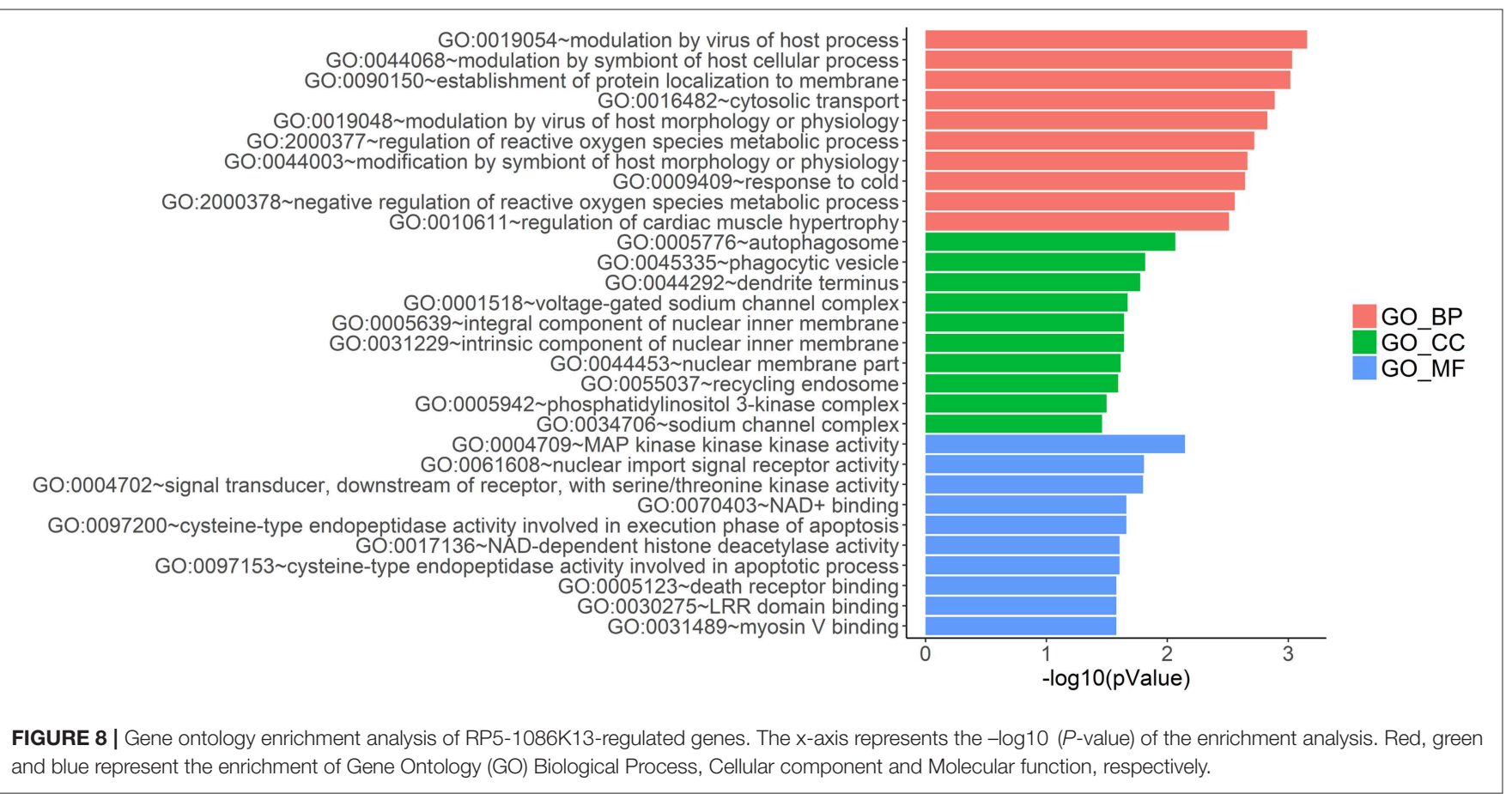

8). Autophagy is crucial for aggressive tumor growth, and this process is deregulated in WT patients $(3,44,45)$. Cadherin belongs to a transmembrane superfamily of proteins, and E-cadherin is used for the diagnosis and prognosis of epithelial cancers (46). Decreased E-cadherin expression has been shown to correlate with a higher stage of WT $(47,48)$. On the other hand, the MAPK pathway is involved in numerous biological processes, including immunity, cell proliferation and tumor-related events, and it plays an important role in the progression of WT $(49,50)$. The participation of LINC00087 and RP5-1086K13 in these biological processes indicated that they have key roles in the progression of WT. However, further 

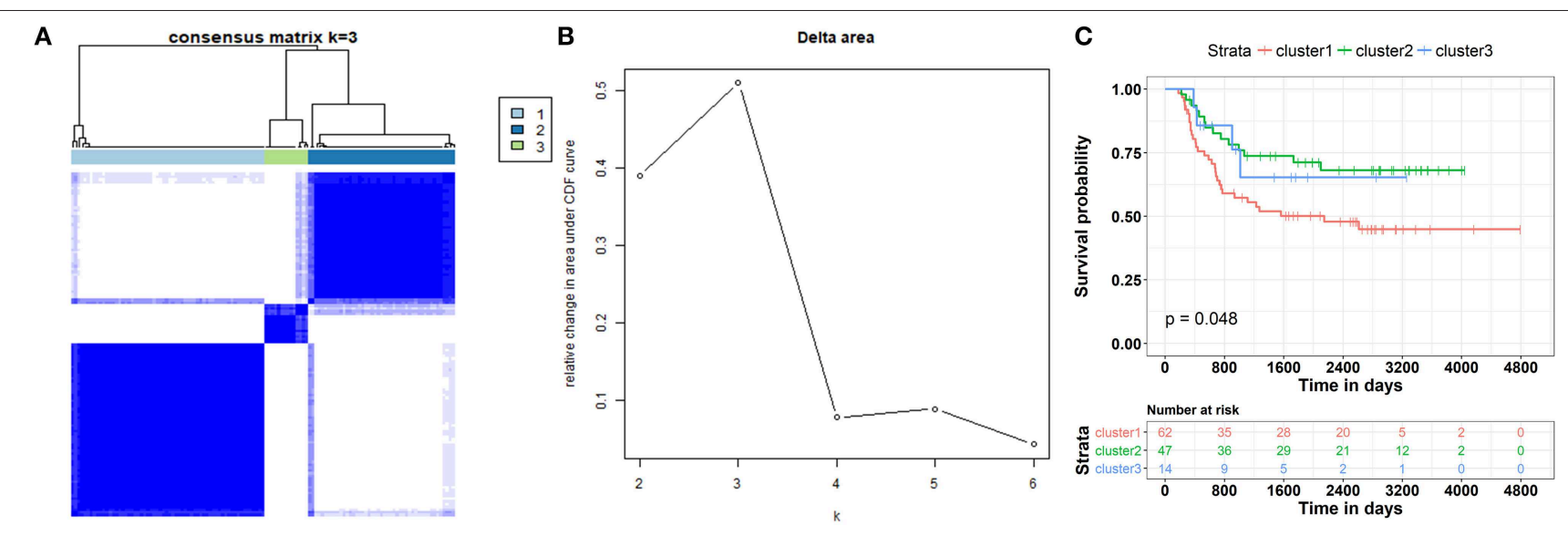

FIGURE 9 | Patient stratification based on clinically relevant ceRNA network. (A) Consensus matrices represented as heatmaps for the chosen optimal cluster number $(k=3)$ for the WT patients. Patient samples are both rows and columns, and consensus values range from 0 to 1 . The dendrogram above the heatmap illustrates the ordering of patient samples in 3 clusters. (B) Corresponding relative change in area under the cumulative distribution function (CDF) curves when cluster number changing from $\mathrm{k}$ to $\mathrm{k}+1$. The range of $\mathrm{k}$ changed from 2 to 10 , and the optimal $k=3$. (C) Kaplan-Meier curve analysis of the overall survival rate in $\mathrm{WT}$ patients at different clusters.

studies are needed to validate the precise mechanisms. The identification of prognostic biomarkers in the ceRNA network pointed to the potential relationship between the network and the clinical outcome of patients. We classified the patients into three categories based on this network (Figure 9). A remarkable correlation was observed between the staging of patients and survival.

Although we have integrated multiple omics data in this study and tried to construct a reliable and clinically significant ceRNA network for the study of prognosis and classification of WT patients, some limitations are still worth noting. First and foremost, as a rare tumor, WT has relatively scarce clinical samples, which lead to fewer omics data for analysis, which may to some extent improve the noise of omics data and reduce the reliability of the whole analysis. In addition, the scarcity of data will increase the difficulty of the found that clinical samples potential rules and patterns, and makes the extracting potentially meaningful and biologically relevant information more difficult. Finally, this research mainly focuses on the use of bioinformatics tools mining WT patients deterioration of underlying mechanisms and biomarker, the results remain to be further investigated in vitro and in vivo. There is reason to believe that in the near future, along with the accumulation of WT clinical and omics data, various experimental methods to further improve, partly as a result will be confirmed in this article, and may be directly used for clinical purposes. In summary, our results showed that the ceRNA network enhanced the molecular staging of cancer patients. Thus, it may facilitate the development of accurate treatment strategies for patients.

\section{DATA AVAILABILITY STATEMENT}

The datasets analyzed during the current study are available in the TARGET repository (https://ocg.cancer.gov/programs/ target/data-matrix).

\section{AUTHOR CONTRIBUTIONS}

JW and FL: conceptualization and writing-review and editing. YW and LH: data curation. LH and MS: formal analysis. YW and $\mathrm{CH}$ : investigation. $\mathrm{LH}$ and $\mathrm{CH}$ : methodology. JW: project administration. MS: resources. FL: supervision. JW: writingoriginal draft.

\section{FUNDING}

This work was supported by the Science and Technology Profect of Shenzhen (Nos. GJHZ20170310090257380, JCYJ20170413092711058, and JCYJ20180228164407689), the China Postdoctoral Science Foundation (No. 2018M631046) and the Scientific Research Program Funded by Shaanxi Provincial Education Department (No. 18JK0979).

\section{SUPPLEMENTARY MATERIAL}

The Supplementary Material for this article can be found online at: https://www.frontiersin.org/articles/10.3389/fonc. 2020.00444/full\#supplementary-material

Figure S1 | Analysis pipeline of the research.

Figure S2 | The pathway terms (from MsigDB) with the most significant p-values.

Figure S3 | The immunologic terms (from MsigDB) with the most significant $p$-values. The $x$-axis represents the number or gene ratio of core module mRNAs involved in the enrichment terms.

Table S1 | The differentially expressed RNAs.

Table S2 | Differentially expressed miRNAs.

Table S3 | The complete list of candidate molecules of ceRNA network.

Table S4 | GO function and pathway enrichment results of candidate genes.

Table S5 | List of the selected target of miRNA.

Table S6 | 103 nodes and 330 edges of the ceRNA network.

Table S7 | Intergrated ceRNA list. 


\section{REFERENCES}

1. Gooskens SL, Segers H, Pritchard-Jones K, Graf N, Dome JS, van den HeuvelEibrink MM. The clinical relevance of age at presentation in nephroblastoma. In: van den Heuvel-Eibrink MM, editor. Wilms Tumor. Brisbane, QLD: Codon Publications (2016).

2. Kanyamuhunga A, Tuyisenge L, Stefan DC. Treating childhood cancer in Rwanda: the nephroblastoma example. Pan Afr Med J. (2015) 21:326. doi: 10.11604/pamj.2015.21.326.5912

3. Li LJ, Wang YL, Yuan LQ, Gu WZ, Zhu K, Yang M, et al. Autophagy inhibition in childhood nephroblastoma and the therapeutic significance. Curr Cancer Drug Targets. (2018) 18:295-303.

4. Furtwangler R, Schmolze M, Graber S, Leuschner I, Amann G, Schenk JP, et al. Pretreatment for bilateral nephroblastomatosis is an independent risk factor for progressive disease in patients with stage V nephroblastoma. Klin Padiatr. (2014) 226:175-81. doi: 10.1055/s-0034-1371840

5. Illhardt T, Ebinger M, Schwarze CP, Feuchtinger T, Furtwangler R, Schlegel PG, et al. Children with relapsed or refractory nephroblastoma: favorable long-term survival after high-dose chemotherapy and autologous stem cell transplantation. Klin Padiatr. (2014) 226:351-6. doi: 10.1055/s-0034-1390504

6. van Waas $M$, Wijnen $M$, Hartman A, de Vries AC, Pieters R, Neggers SJ, et al. Daily life physical activity in long-term survivors of nephroblastoma and neuroblastoma. J Pediatr Hematol Oncol. (2013) 35:361-5. doi: 10.1097/MPH.0b013e31827e8fb9

7. Boutet A, Comai G, Schedl A. The WTX/AMER1 gene family: evolution, signature and function. BMC Evol Biol. (2010) 10:280. doi: 10.1186/1471-2148-10-280

8. Wang X, Gao P, Lin F, Long M, Weng Y, Ouyang Y, et al. Wilms' tumour suppressor gene 1 (WT1) is involved in the carcinogenesis of lung cancer through interaction with PI3K/Akt pathway. Cancer Cell Int. (2013) 13:114. doi: 10.1186/1475-2867-13-114

9. Wang H, Zhou H, Liu A, Guo X, Yang CS. Genetic analysis of colon tumors induced by a dietary carcinogen PhIP in CYP1A humanized mice: identification of mutation of beta-catenin/Ctnnb1 as the driver gene for the carcinogenesis. Mol Carcinog. (2015) 54:1264-74. doi: 10.1002/mc.22199

10. Rui Y, Hu M, Wang P, Zhang C, Xu H, Li Y, et al. LncRNA HOTTIP mediated DKK1 downregulation confers metastasis and invasion in colorectal cancer cells. Histol Histopathol. (2018) 34:619-30. doi: 10.14670/HH-18-043

11. Tian Y, Sun C, Zhang L, Pan Y. Clinical significance of miRNA $106 \mathrm{a}$ in non-small cell lung cancer patients who received cisplatin combined with gemcitabine chemotherapy. Cancer Biol Med. (2018) 15:15764. doi: 10.20892/j.issn.2095-3941.2017.0182

12. de Sa Pereira BM, Azevedo RM, Aguirre Neto JC, Menezes CF, Rodrigues KE, Faria PA, et al. Intra-tumor genetic heterogeneity in Wilms tumor samples. Rev Assoc Med Bras. (2019) 65:1496-501. doi: 10.1590/1806-9282.65.12.1496

13. Gong Y, Zou B, Chen J, Ding L, Li P, Chen J, et al. Potential five-MicroRNA signature model for the prediction of prognosis in patients with wilms tumor. Med Sci Monit. (2019) 25:5435-44. doi: 10.12659/MSM.916230

14. Ludwig N, Werner TV, Backes C, Trampert P, Gessler M, Keller A, et al. Combining miRNA and mRNA expression profiles in wilms tumor subtypes. Int J Mol Sci. (2016) 17:475. doi: 10.3390/ijms17040475

15. Rubio-Somoza I, Weigel D, Franco-Zorilla JM, Garcia JA, PazAres J. ceRNAs: miRNA target mimic mimics. Cell. (2011) 147:1431-2. doi: 10.1016/j.cell.2011.12.003

16. Zhang Z, Qian W, Wang S, Ji D, Wang Q, Li J, et al. Analysis of lncRNA-associated ceRNA network reveals potential lncRNA biomarkers in human colon adenocarcinoma. Cell Physiol Biochem. (2018) 49:177891. doi: 10.1159/000493623

17. Lian Y, Xiong F, Yang L, Bo H, Gong Z, Wang Y, et al. Long noncoding RNA AFAP1-AS1 acts as a competing endogenous RNA of miR423-5p to facilitate nasopharyngeal carcinoma metastasis through regulating the Rho/Rac pathway. J Exp Clin Cancer Res. (2018) 37:253. doi: 10.1186/s13046-018-0918-9

18. Wang Q, Cai J, Fang C, Yang C, Zhou J, Tan Y, et al. Mesenchymal glioblastoma constitutes a major ceRNA signature in the TGF-beta pathway. Theranostics. (2018) 8:4733-49. doi: 10.7150/thno.26550

19. Fang XN, Yin M, Li H, Liang C, Xu C, Yang GW, et al. Comprehensive analysis of competitive endogenous RNAs network associated with head and neck squamous cell carcinoma. Sci Rep. (2018) 8:10544. doi: 10.1038/s41598-018-28957-y

20. Do D, Bozdag S. Cancerin: a computational pipeline to infer cancerassociated ceRNA interaction networks. PLoS Comput Biol. (2018) 14:e1006318. doi: 10.1371/journal.pcbi.1006318

21. Song J, Peng J, Zhu C, Bai G, Liu Y, Zhu J, et al. Identification and validation of two novel prognostic lncRNAs in kidney renal clear cell carcinoma. Cell Physiol Biochem. (2018) 48:2549-62. doi: 10.1159/000492699

22. Langfelder $P$, Horvath S. WGCNA: an $R$ package for weighted correlation network analysis. BMC Bioinform. (2008) 9:559. doi: 10.1186/1471-2105-9-559

23. Feichtinger RG, Neureiter D, Royer-Pokora B, Mayr JA, Zimmermann FA, Jones N, et al. Heterogeneity of mitochondrial energy metabolism in classical triphasic wilms' tumor. Front Biosci. (2011) 3:187-93. doi: 10.2741/e232

24. Wegert J, Vokuhl C, Ziegler B, Ernestus K, Leuschner I, Furtwangler R, et al. TP53 alterations in wilms tumour represent progression events with strong intratumour heterogeneity that are closely linked but not limited to anaplasia. J Pathol Clin Res. (2017) 3:234-48. doi: 10.1002/cjp2.77

25. Li H, Zhu Y, Burnside ES, Drukker K, Hoadley KA, Fan C, et al. MR imaging radiomics signatures for predicting the risk of breast cancer recurrence as given by research versions of mammaprint, oncotype DX, and PAM50 gene assays. Radiology. (2016) 281:382-91. doi: 10.1148/radiol.2016152110

26. Godec J, Tan Y, Liberzon A, Tamayo P, Bhattacharya S, Butte AJ, et al. Compendium of immune signatures identifies conserved and speciesspecific biology in response to inflammation. Immunity. (2016) 44:194206. doi: 10.1016/j.immuni.2015.12.006

27. Xue B, Oldfield CJ, Dunker AK, Uversky VN. CDF it all: consensus prediction of intrinsically disordered proteins based on various cumulative distribution functions. FEBS Lett. (2009) 583:1469-74. doi: 10.1016/j.febslet.2009.03.070

28. Monti S, Tamayo P, Mesirov J, Golub T. Consensus clustering: a resampling based method for class discovery and visualization of gene expression microarray data. Machine Learn. (2003) 52:91-118. doi: 10.1023/A:1023949509487

29. Cone EB, Dalton SS, Van Noord M, Tracy ET, Rice HE, Routh JC. Biomarkers for wilms tumor: a systematic review. J Urol. (2016) 196:15305. doi: 10.1016/j.juro.2016.05.100

30. Meng M, Li L, Li R, Wang W, Chen Y, Xie Y, et al. A dynamic transcriptomic atlas of cytokine-induced killer cells. J Biol Chem. (2018) 293:1960012. doi: $10.1074 /$ jbc.RA118.003280

31. Guo YZ, Sun HH, Wang XT, Wang MT. Transcriptomic analysis reveals key lncRNAs associated with ribosomal biogenesis and epidermis differentiation in head and neck squamous cell carcinoma. J Zhejiang Univ Sci B. (2018) 19:674-88. doi: 10.1631/jzus.B1700319

32. Di Y, Chen D, Yu W, Yan L. Bladder cancer stage-associated hub genes revealed by WGCNA co-expression network analysis. Hereditas. (2019) 156:7. doi: 10.1186/s41065-019-0083-y

33. Ding M, Li F, Wang B, Chi G, Liu H. A comprehensive analysis of WGCNA and serum metabolomics manifests the lung cancerassociated disordered glucose metabolism. I Cell Biochem. (2019) 120:10855-63. doi: 10.1002/jcb.28377

34. Wan Q, Tang J, Han Y, Wang D. Co-expression modules construction by WGCNA and identify potential prognostic markers of uveal melanoma. Exp Eye Res. (2018) 166:13-20. doi: 10.1016/j.exer.2017.10.007

35. Yang Q, Wang R, Wei B, Peng C, Wang L, Hu G, et al. Candidate Biomarkers and molecular mechanism investigation for glioblastoma multiforme utilizing WGCNA. Biomed Res Int. (2018) 2018:4246703. doi: 10.1155/2018/4246703

36. Wang $\mathrm{X}$, Song $\mathrm{P}$, Huang $\mathrm{C}$, Yuan $\mathrm{N}$, Zhao $\mathrm{X}$, Xu C. Weighted gene co-expression network analysis for identifying hub genes in association with prognosis in Wilms tumor. Mol Med Rep. (2019) 19:2041-50. doi: 10.3892/mmr.2019.9881

37. Liu S, Chen S, Ma K, Shao Z. Prognostic value of kindlin-2 expression in patients with solid tumors: a meta-analysis. Cancer Cell Int. (2018) 18:166. doi: 10.1186/s12935-018-0651-7

38. Balmana M, Mereiter S, Diniz F, Feijao T, Barrias CC, Reis CA. Multicellular human gastric-cancer spheroids mimic the glycosylation phenotype of gastric carcinomas. Molecules. (2018) 23:2815. doi: 10.3390/molecules23112815

39. Yap LW, Brok J, Pritchard-Jones K. Role of CD56 in normal kidney development and wilms tumorigenesis. Fetal 
Pediatr Pathol. (2017) 36:62-75. doi: 10.1080/15513815.2016.12 56358

40. Hu Q, Gao F, Tian W, Ruteshouser EC, Wang Y, Lazar A, et al. Wt1 ablation and Igf2 upregulation in mice result in wilms tumors with elevated ERK1/2 phosphorylation. J Clin Invest. (2011) 121:174-83. doi: 10.1172/JCI43772

41. Schoenhals JE, Cushman TR, Barsoumian HB, Li A, Cadena AP, Niknam S, et al. Anti-glucocorticoid-induced tumor necrosis factorrelated protein (GITR) therapy overcomes radiation-induced treg immunosuppression and drives abscopal effects. Front Immunol. (2018) 9:2170. doi: 10.3389/fimmu.2018.02170

42. Xue X, Wang X, Zhao Y, Hu R, Qin L. Exosomal miR-93 promotes proliferation invasion in hepatocellular carcinoma by directly inhibiting TIMP2/TP53INP1/CDKN1A. Biochem Biophys Res Commun. (2018) 502:515-21. doi: 10.1016/j.bbrc.2018.05.208

43. Li HY, Liang JL, Kuo YL, Lee HH, Calkins MJ, Chang HT, et al. miR105/93-3p promotes chemoresistance and circulating miR-105/93-3p acts as a diagnostic biomarker for triple negative breast cancer. Breast Cancer Res. (2017) 19:133. doi: 10.1186/s13058-017-0918-2

44. Guimei M, Eladl MA, Ranade AV, Manzoor S. Autophagy related markers (Beclin-1 and ATG4B) are strongly expressed in wilms' tumor and correlate with favorable histology. Histol Histopathol. (2018) 34:4756. doi: 10.14670/HH-18-023

45. Hu M, Luo Q, Alitongbieke G, Chong S, Xu C, Xie L, et al. CelastrolInduced Nur77 interaction with TRAF2 alleviates inflammation by promoting mitochondrial ubiquitination and autophagy. Mol Cell. (2017) 66:141-53. e146. doi: 10.1016/j.molcel.2017.03.008

46. Baudry D, Cabanis MO, Patte C, Zucker JM, Pein F, Fournet JC, et al. Cadherins in wilms' tumor: E-cadherin expression despite absence of WT1. Anticancer Res. (2003) 23:475-8.
47. Safford SD, Freemerman AJ, Langdon S, Bentley R, Goyeau D, Grundy $\mathrm{PE}$, et al. Decreased E-cadherin expression correlates with higher stage of wilms' tumors. J Pediatr Surg. (2005) 40:341-8. doi: 10.1016/j.jpedsurg.2004. 10.030

48. van Roy F. Beyond E-cadherin: roles of other cadherin superfamily members in cancer. Nat Rev Cancer. (2014) 14:121-34. doi: 10.1038/nr c3647

49. Eleveld TF, Schild L, Koster J, Zwijnenburg DA, Alles LK, Ebus ME, et al. RAS-MAPK pathway-driven tumor progression is associated with loss of CIC and other genomic aberrations in neuroblastoma. Cancer Res. (2018) 78:6297-307. doi: 10.1158/0008-5472.CAN-181045

50. Mine A, Berens ML, Nobori T, Anver S, Fukumoto K, Winkelmuller TM, et al. Pathogen exploitation of an abscisic acid- and jasmonateinducible MAPK phosphatase and its interception by arabidopsis immunity. Proc Natl Acad Sci USA. (2017) 114:7456-61. doi: 10.1073/pnas.17026 13114

Conflict of Interest: The authors declare that the research was conducted in the absence of any commercial or financial relationships that could be construed as a potential conflict of interest.

Copyright (c) 2020 Wang, Wang, Han, Shahen, Hu and Li. This is an open-access article distributed under the terms of the Creative Commons Attribution License (CC BY). The use, distribution or reproduction in other forums is permitted, provided the original author(s) and the copyright owner(s) are credited and that the original publication in this journal is cited, in accordance with accepted academic practice. No use, distribution or reproduction is permitted which does not comply with these terms. 